\title{
Subject Index Vol. 28, 1996
}

$\mathrm{N}$-Acetyl aspartyl glutamic acid 80 Acid phosphatase 141 After-cataract 201 Aging 160,343 Alcohol 237 Amino acids 319 3Aminotriazole 336 Angiogenin 356 Animal(s) 130 model 19 Anisometropia 137 Anterior chamber 176

- fluid 361

- chamber-associated immune

deviation 289

eye segment 265 Antimitotic drugs 64 Antioxidant(s) 171, 184 enzymes 336 Aqueous flare 147,260 Aspirin 108 Attachment 201 Australia 88 Autofluorescence 160 Autoregulation 343

Blood-aqueous barrier 137 Bovine 141, 184

- $\quad$ lens 365

Catalase 8,331,336 Cataract 88, 209, 224, 237, 265, 275

surgery 64 Cell adhesion 2

culture $50,153,165,171$

surface structure 153

viability 153 Central retinal artery 193 Chang's conjunctival cells 153 Chaperone activity 365

Choline 319

Choroid 36,255

Collagen 165

Color Doppler imaging 193

Cone photoreceptor cells 117 Conjunctiva 130 Contrast sensitivity 193 Conventional tonography 312 Cornea 50,71, 130, 141,319 Cross-linking structure 29 Crystallins 230 Cyclic guanosine 3',5'-monophos-

phate $19---$ phosphodiesterase 19 Cytokine 248 Cytotoxicity 153

Dapiprazole 312 Decreased reaction 147 Delayed-type hypersensitivity 289 Development 270

Diabetes 57,71,275 Diabetic cataracts 230 Digital image processing 265 DNA histogram 153

Docosahexanoic acid 93

Electron microscopy 71 Elschnigspot 255 Enzyme-linked immunosorbent assay 356 Epithelium 50,71 ERG campimetry 303 Ethnicity 88

Fatty acids 93

Fibrin 176

Fibronectin 201

Fine structure 36

Flare density 137

Flow cytometry 64

Fluorophotometry 160

5-Fluorouracil 64

Focal electroretinogram 303 
Frozen storage 219

Frozen-sectioning technique 365

Fructose-3-phosphate 57

Glaucoma 351

Glial fibrillary acidic protein 99

Glucose 275

- $\quad$ metabolism 57

Glycation 230

Growth factors 361

Guinea pig 29

Heat shock protein 22 270, 284 Heparan sulphate 176 Herpes simplex virus type 1 125, 289

- $\quad$ - - type 2289

Hexosaminidase 141

High myopia 137

High-resolution NMR spectros-

copy $319 \mathrm{HM}-, \alpha \mathrm{L}-, \beta \mathrm{H}-, \beta \mathrm{L}-, \beta \mathrm{s}-,, \gamma$-Crystal-

lins 365 Host range 125 Human embryo 270 Hypertension 255 Hypotaurine 319

Immunohistochemistry 270, 296 Impression cytology 71 In situ hybridisation 296 Interferon 125

Interferon- $\gamma 248$ Interleukin-6 248 Interphotoreceptor matrix 117 Intraocular pressure 351

Ischemia 331 Ischemia-reperfusion 248 Isoelectric focusing 365

Keratopathy 71

Labrador retriever 19 Lactate dehydrogenase 141 Laser Doppler technique 108,343 Lectin-

binding properties 117 Lens 160,209,270,275

epithelial cells 201 Leucine arylamidase 141 7/8Leucine tracing 36 Light 99

scattering 209

377

Linoleic acid 8

- hydroperoxide 8 Lipid peroxidation 184

peroxide 336 Lodoxamide 80 Lysosomal enzymes 141

Man 130 Mature rat lens 57 Meibomian gland secretion 44 Metabolites 319 Microaneurysm 255

Miotics 312 MIP26 glycation 230 MitomycinC 64, 153 Mouse 50 Myotonic dystrophy 224

- $\quad$ - mutation 224

Near ultraviolet 2

Nitric oxide synthase inhibitor

260 NMR spectroscopy 57

Ocular effects 351

infection 125

neovascularization 356 Ophthalmic artery 193 Opsin 296

Optic nerve axon 29 Oxidation 275

31P nuclear magnetic resonance

stectroscopy 44 Parinaric acid 275 Peanut agglutinin 117 Pentifylline 165 Pentoxifylline 165

Phospholipids 44, 93 Photopic electroretinogram 303 Photoreceptor damage 99 Pigmentary

clearance 312

- glaucoma 312

Pigmented rabbits, aqueous flare

260 
Pineal 99

Porcine 184

Proliferative diabetic retinopathy

356 Prostaglandin(s) 260

E2 147

F2 $\alpha 351$

Protein degradation 219 Proton NMR spectrosocpy 319

Quick-freezing, deep-etching method 29

Rabbit 176 Rat 209

lens 219

retina 248 Receptors 361 Red blood cells 93 Regenerating protein 130 Relative risk ratio 88

Repeated application 147 Reperfusion 331

Retina 99,117, 184,296,331

-, guinea pig 336

- , rabbit 336

- , rat 336

Retinal arterial blood speed 108

blood flow 108,343

dystrophy 19

perfusion pressure 343

pigment epithelial cells 8

- epithelium 2, 184

vessel diameter 343 Retinitis pigmentosa 93 Reverse transcription-polymerase

chain reaction 248 RGDSpeptide 201 Rhodopsin 296 Risk factors 237 Rod outer segments 184

Saponin 29

Scheimpflug photography 230, 265

Secondary cataract 64 Slitlamp 265

- $\quad$ examination 224

Smoking 237

Sorbitol-3-phosphate 57

Superior cervical ganglion 36

Superoxide dismutase 8

Sympathetic innervation 36

Tachyphylaxis 147

Tear film 44

Tenon's capsule fibroblast(s) 165 ,

171 Therapy 80 Thymidine kinase $125 \mathrm{~J}$ - $\alpha$-Tocopherol 171 Topical application 351

Trabeculectomy 171 Transforming growth factor- $\alpha 361$

- $\quad$ - factor- $\beta$ i 248

Tree shrew 160

Trypsin resistance 2

Tumor necrosis factor 248

Type I diabetic patients 108

Tyrosine hydroxylase immunohis-

tochemistry 36

Ultrastructure 19,117 Ultraviolet radiation 88 
- $\quad$-, in vivo 209

Uveitis 284

Variance 141

Vernal keratoconjunctivitis 80

Vimentin 219

Visual acuity 193

- $\quad$ field 303

Vitamin E 171

Water-soluble/-insoluble crystal-

lins 365 Wound healing 361

Yersinia enterocolitica 284

378

Subject Index Vol. 28, 1996 